DESY 94-148

hep-ph/9409457

August 1994

\title{
Bounds on Radil and Magnetic Dipole Moments OF QuARKS AND LePtons FROM LEP, SLC AND HERA
}

\author{
G. Köpp ${ }^{1}$, D. Schaile², M. Spira ${ }^{3}$ and P.M. Zerwas ${ }^{3}$ \\ ${ }^{1}$ IIIA Phys. Institut, RWTH, D-52056 Aachen, FRG \\ 2 CERN, CH-1211 Geneva 23, Switzerland \\ ${ }^{3}$ Deutsches Elektronen-Synchrotron DESY, D-22603 Hamburg, FRG
}

\begin{abstract}
Leptons, quarks and gauge bosons are assumed to be pointlike particles in the Standard Model. Stringent bounds on the radii of quarks and leptons and their weak anomalous magnetic moments can be derived from the high-precision measurements at LEP and SLC. We find a model-independent bound of $R \lesssim 10^{-17} \mathrm{~cm}$ for quark and lepton radii. HERA will provide complementary information on the electromagnetic static properties of the quarks and the parameters of the charged quark currents.
\end{abstract}




\section{INTRODUCTION}

The Standard Model has proven so tremendously sucessful that any studies of the terra incognita beyond are highly speculative. However, the Higgs sector allows conclusions on possible boundaries of the model. If the Higgs mass is light, the model can be extrapolated to energy scales of the order of the Planck mass, yet likely demanding a supersymmetric extension in the $\mathrm{TeV}$ range. Also, if the Higgs mass is heavy, new physical phenomena may emerge at energy scales in the $\mathrm{TeV}$ range.

The fundamental particles - leptons and quarks, gauge and Higgs bosons - are assumed to be pointlike in the Standard Model. Possible substructures as well as any new interactions at high energies would manifest themselves as non-zero radii and anomalous moments of these particles.

Deep-inelastic scattering of electrons on protons is one of the classical tools to probe the static parameters of quarks [1]. This method was based originally on the assumption that the photon is elementary and that electrons can be treated effectively as pointlike particles down to distances of order $10^{-17} \mathrm{~cm}$. Supporting evidence for this assumption follows from the high-precision measurements of the magnetic dipole moment of the electron [2]. If non-standard contributions to the anomalous magnetic moment, $\delta(g-2)_{e} \lesssim 10^{-10}$, scale linearly with the fermion mass, the electron radius is bound to $R_{e} \lesssim 2 \cdot 10^{-21} \mathrm{~cm}$. However, if $\delta(g-2)_{e}$ depends quadratically on the fermion mass the bound is weakened to $R_{e} \lesssim 3 \cdot 10^{-16} \mathrm{~cm}$ and non-pointlike substructures of the electrons have to be taken into account eventually when deep-inelastic scattering data are evaluated. The quadratic mass dependence is a natural consequence of chiral symmetry [3] which could keep the fermion masses much smaller than the energy scale $R^{-1}$ of the substructure and could allow for non-zero axial couplings for small $R$ [1]. The radius of the muon is smaller by an order of magnitude in this scenario.

Complementary information can be extracted from $e^{+} e^{-}$annihilation into fermion pairs, in particular on the $Z$ resonance. While the assumption of elementary photons may be considered quite natural, the analogous assumption for the massive $Z$ boson is less obvious; note, however, that magnetic and quadrupole moments of a composite spinone system have been shown [5] to approach the standard-model values in the zero-radius limit. Possible deviations of the $Z \bar{F} F$ vertex from the pointlike form may therefore be attributed to substructures either of the quarks or of the $Z$ boson. However, barring unnatural cancellations, bounds derived from the data will apply approximately to both species of particles at the same time.

Setting bounds on anomalous values of the electroweak static parameters provides an alternative method to the analysis of contact interactions in $e e, e q$ and $q q$ elastic scattering [6]. The bounds on the energy scales $\Lambda$ of the contact interactions, typically about 2 to $5 \mathrm{TeV}$ for electrons [7] and above $1 \mathrm{TeV}$ for quarks [8], can be transformed into bounds on the radii of electrons and quarks only after the new strong coupling $g_{*}$ is 
fixed, $R \sim \sqrt{4 \pi / g_{*}^{2}} \Lambda^{-1}$. $\Lambda$ values of more than $1 \mathrm{TeV}$ correspond to radii $R \lesssim 6 \times 10^{-17} \mathrm{~cm}$ within factors of three.

Assuming $\mathcal{C}, \mathcal{P}$ invariance for the $\gamma$ couplings to fermions and $\mathcal{C P}$ invariance for the $Z, W$ couplings, the $\gamma, Z, W$-fermion vertices ${ }^{\Uparrow} \Gamma_{\lambda}$ are parameterized by form factors, depending on the momentum transfer $q$, in the following way:

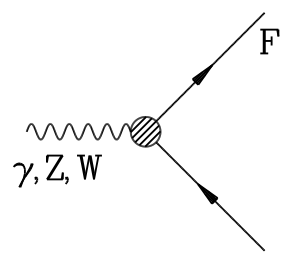

$$
\begin{aligned}
\Gamma_{\lambda}^{\gamma} & =e_{0} e\left[f \gamma_{\lambda}+i \frac{\kappa}{2 m_{F}} \sigma_{\lambda \rho} q_{\rho}\right] \\
\Gamma_{\lambda}^{Z} & =\sqrt{\frac{G_{F} m_{Z}^{2}}{2 \sqrt{2}}}\left[v f \gamma_{\lambda}+i \frac{\kappa}{2 m_{F}} v \sigma_{\lambda \rho} q_{\rho}-a f \gamma_{\lambda} \gamma_{5}\right] \\
\Gamma_{\lambda}^{W} & =\sqrt{\frac{G_{F} m_{W}^{2}}{\sqrt{2}}}\left(1+\gamma_{5}\right)\left[f \gamma_{\lambda}+i \frac{\kappa}{2 m_{F}} \sigma_{\lambda \rho} q_{\rho}\right]
\end{aligned}
$$

The form factors $f$ reduce to unity and the couplings to the familiar values in the Standard Model $\left[e_{0} e ; v=2 I_{3}-4 e \sin ^{2} \theta_{w}\right.$ and $\left.a=2 I_{3}\right]$ in the pointlike limit. For the $\mathcal{C} \mathcal{C}$ processes we take the neutrinos in the final state as purely left-handed particles [and the quarks, too]. For the sake of simplicity we also assume the radius $R$ to be universal for the weak vector and axial-vector probesi so that the form factor is parameterized in the standard way as

$$
f=1+\frac{1}{6} R^{2} q^{2}
$$

$\kappa$ is the anomalous magnetic dipole moment of the fermion $F$ in units of the $F$ Bohr magneton $e_{0} e / 2 m_{F}$ etc. For light $(u, d)$ and $s$ quarks we identify $m_{q}$ with the values $\approx 4$ $\mathrm{MeV}$ and $95 \mathrm{MeV}$, respectively, corresponding to the running current quark masses defined at the scale $m_{Z}$; for charm and bottom quarks we choose 0.7 and $3.0 \mathrm{GeV}$ [11]. For complex systems the magnitude of the anomalous magnetic dipole couplings is expected to be of the spatial size of the system [3] so that $\kappa$ should scale with the $F$ mass. Since dipole

\begin{tabular}{|c|cc|}
\hline & $\begin{array}{c}\text { linear } F \text { mass } \\
\text { dependence }\end{array}$ & $\begin{array}{c}\text { quadratic } F \text { mass } \\
\text { dependence }\end{array}$ \\
\hline \hline$\kappa$ & $\eta_{1} m_{F} R$ & $\left(\eta_{2} m_{F} R\right)^{2}$ \\
$\frac{\kappa}{2 m_{F}}$ & $\frac{1}{2} \eta_{1} R$ & $\frac{1}{2} m_{F}\left(\eta_{2} R\right)^{2}$ \\
\hline
\end{tabular}

Table 1: Definition of the dimensionless parameters $\eta_{1}$ and $\eta_{2}$, assumed to be independent of the fermion mass.

${ }^{*} \mathcal{C P}$ non-invariance of these interactions gives rise to electric dipole moments which have recently attracted theoretical [9] and experimental attention [10].

$\dagger$ The form factors $f$ and the anomalous magnetic moments may depend, in principle, on the vector bosons $\gamma, Z, W$ as well as on the fermion species. 
interactions flip the chirality of the states, $\kappa$ will scale quadratically with the $F$ mass in chirally symmetric theories [3]. Particularly in the latter scenario, the heavy fermions $\tau$, $c, b$ [and $t$ in the future [12]] are of special interest since the anomalous contributions are enhanced by seven to eight orders of magnitude over the electron parameters. For the linear and quadratic $F$ mass scenarios we therefore define two parameters $\eta_{1}$ and $\eta_{2}$, as shown in Table 1, which are assumed to be independent of the fermion $F$ mass. The bounds derived for the electron radius $R_{e}$ from the measurements of the anomalous magnetic moment $\kappa_{e}$ correspond to the values $\eta_{1,2}=1$ by definition. In high-energy scattering experiments the particle radius $R$ and the anomalous magnetic dipole moments can be measured separately so that these two independent parameters can be disentangled.

\section{2. $e^{+} e^{-}$AnNihilation AND $Z$ DeCAys}

The cross section for the process $e^{-}+e^{+} \rightarrow F+\bar{F}$ is mediated by $s$-channel $\gamma$ and $Z$ boson exchange for $F \neq e$. Neglecting the fermion masses in a first step [see Ref. 12, 13. for mass corrections], the cross section can be written as an incoherent superposition of the helicity cross sections $\sigma\left(e_{i}^{-}+e_{j}^{+} \rightarrow F_{k}+\bar{F}_{l}\right)$ with $i, \ldots=L, R$ :

$$
\frac{d \sigma}{d \cos \theta}=\frac{4 \pi \alpha^{2} N_{\mathcal{C}}}{3 s} \frac{1}{4} \sum_{L, R} \sigma_{i j}^{k l} f_{i j}^{k l}(\cos \theta)
$$

$s$ denotes the total energy squared and $\theta$ the angle between the $F$ momentum and the $e^{-}$beam axis; $N_{\mathcal{C}}=3$ and 1 for quark and leptons, respectively. Vector/axial-vector currents $[V]$ are helicity conserving while tensor currents $[\Sigma]$ involving the anomalous magnetic moments, flip the helicity so that we obtain the following angular distributions for the annihilation/creation of fermion-antifermion pairs:

$$
f_{i j}^{k l}(\cos \theta)= \begin{cases}\frac{3}{8}(1 \pm \cos \theta)^{2} & \text { for } V_{e} \times V_{F}\left[\operatorname{hel}(F)= \pm \operatorname{hel}\left(e^{-}\right)\right] \\ \frac{3}{8} \sin ^{2} \theta & \text { for } V_{e} \times \Sigma_{F} \text { and } v . v . \\ \frac{3}{8} \cos ^{2} \theta & \text { for } \Sigma_{e} \times \Sigma_{F}\end{cases}
$$

The form of these coefficients can easily be derived from angular momentum conservation. Since vector/axial-vector currents couple the particles to spin-1 states polarized along the flight direction, either backward or forward production is forbidden [equal and opposite helicities in the initial and final states]. Since the spin currents $\Sigma$ couple the particles to spin $S_{z}=0$ states, the spin of the intermediate $\gamma, Z$ bosons points into the direction perpendicular to the fermion flight axes so that $F$ production at $90^{\circ}$ cannot occur in this case. On the other hand, angular momentum conservation forbids non-zero values of the $V \times \Sigma$ interference terms for forward and backward production since the spin $S_{z}$ would change along the beam axis by one unit without being balanced by orbital angular momenta. 
Introducing the generalized charges, related to vector/axial-vector and spin currents,

$$
\begin{array}{rlr}
Q_{i}^{k} & =e_{e} e_{F}+\frac{G_{F} m_{Z}^{2}}{8 \sqrt{2} \pi \alpha} \epsilon_{e}^{i} \epsilon_{F}^{k} \frac{s}{s-m_{Z}^{2}+i m_{Z} \Gamma_{Z}} & \text { for } V_{e} \times V_{F} \\
Q_{i}^{\prime}=e_{e} e_{F}+\frac{G_{F} m_{Z}^{2}}{8 \sqrt{2} \pi \alpha} \epsilon_{e}^{i} v_{F} \frac{s}{s-m_{Z}^{2}+i m_{Z} \Gamma_{Z}} & \text { for } V_{e} \times \Sigma_{F} \\
Q^{\prime k}=e_{e} e_{F}+\frac{G_{F} m_{Z}^{2}}{8 \sqrt{2} \pi \alpha} v_{e} \epsilon_{F}^{k} \frac{s}{s-m_{Z}^{2}+i m_{Z} \Gamma_{Z}} & \text { for } \Sigma_{e} \times V_{F} \\
Q^{\prime \prime}=e_{e} e_{F}+\frac{G_{F} m_{Z}^{2}}{8 \sqrt{2} \pi \alpha} v_{e} v_{F} \frac{s}{s-m_{Z}^{2}+i m_{Z} \Gamma_{Z}} & \text { for } \Sigma_{e} \times \Sigma_{F}
\end{array}
$$

with $\epsilon^{L, R}=v \pm a$, the coefficients $\sigma_{i j}^{k l}$ are given by

$$
\sigma_{i j}^{k l}= \begin{cases}\left|Q_{i}^{k}\right|^{2} f_{e}^{2} f_{F}^{2} & \text { for } V_{e} \times V_{F} \\ \left|Q_{i}^{\prime}\right|^{2} f_{e}^{2} \frac{1}{4}\left(\kappa_{F} / m_{F}\right)^{2} s & \text { for } V_{e} \times \Sigma_{F} \\ \left|Q^{\prime k}\right|^{2} \frac{1}{4}\left(\kappa_{e} / m_{e}\right)^{2} s f_{F}^{2} & \text { for } \Sigma_{e} \times V_{F} \\ \left|Q^{\prime \prime}\right|^{2} \frac{1}{16}\left(\kappa_{e} / m_{e}\right)^{2}\left(\kappa_{F} / m_{F}\right)^{2} s^{2} & \text { for } \Sigma_{e} \times \Sigma_{F}\end{cases}
$$

Non-zero radii and anomalous magnetic moments affect the total cross sections and the angular distributions of the produced fermions.

On top of the $Z$ boson, three observables are of particular interest - the partial width $\Gamma(Z \rightarrow F \bar{F})$, the forward-backward asymmetry of the leptons/quark jets and the $\alpha_{F}$ parameter, defined by $d N / d \cos \theta \sim 1+\alpha_{F} \cos ^{2} \theta+\beta_{F} \cos \theta$ and measuring the strength of the longitudinal cross section. Deviations from the [improved] Born cross sections, marked by the index $B$, may be expressed in terms of the electron and quark/lepton radii $R_{F}$ and the anomalous moments $\kappa_{F}$ in the following way [ $F$ mass terms included]:

$$
\begin{aligned}
\Gamma(Z \rightarrow F \bar{F}) & =\Gamma_{B}(Z \rightarrow F \bar{F})\left[1+\frac{1}{3}\left(m_{Z} R_{F}\right)^{2}+3 \kappa_{1}\right] \\
A_{F B}(F) & =A_{F B}^{B}\left[1+\kappa_{2}\right] \\
\alpha_{F} & =1-4 \kappa_{3}
\end{aligned}
$$

Additional information is provided by the left-right asymmetry for polarized electrons/positrons, as well as the LR asymmetry of the polarized $\tau$ 's in the final state.

$$
\begin{aligned}
& \mathcal{A}_{e}=\mathcal{A}_{e}^{B}\left[1+\kappa_{4}\right] \\
& \mathcal{A}_{\tau}=\mathcal{A}_{\tau}^{B}\left[1+\frac{3}{2} \kappa_{5}\right]
\end{aligned}
$$

${ }^{*}$ Note that $\kappa_{F} / m_{F} \sim R$ or $m_{F} R^{2}$ does not rise with the inverse fermion mass so that the limit $m_{F} \rightarrow 0$ can safely be applied everywhere, in particular for electrons. 
The coefficients $\kappa_{i}$ are given by

$$
\begin{aligned}
& \kappa_{1}=\kappa_{F}\left\{1+\frac{1}{24} \kappa_{F}\left(m_{Z} / m_{F}\right)^{2}\right\} \hat{v}_{q}^{2} \\
& \kappa_{2}=\kappa_{F}\left\{1-3 \hat{v}_{F}^{2}-\frac{1}{8} \kappa_{F}\left(m_{Z} / m_{F}\right)^{2} \hat{v}_{F}^{2}\right\}-\frac{1}{8} \kappa_{e}^{2}\left(m_{Z} / m_{e}\right)^{2} \hat{v}_{e}^{2} \\
& \kappa_{3}=\kappa_{F}\left\{1+\frac{1}{8} \kappa_{F}\left(m_{Z} / m_{F}\right)^{2}\right\} \hat{v}_{F}^{2}+\frac{1}{8} \kappa_{e}^{2}\left(m_{Z} / m_{e}\right)^{2} \hat{v}_{e}^{2}
\end{aligned}
$$

and, for the LR asymmetries,

$$
\begin{aligned}
& \kappa_{4}=-\frac{1}{8} \kappa_{e}^{2}\left(m_{Z} / m_{e}\right)^{2} \hat{v}_{e}^{2} \\
& \kappa_{5}=\kappa_{\tau}\left\{1-4 \hat{v}_{\tau}^{2}-\frac{1}{12} \kappa_{\tau}\left(m_{Z} / m_{\tau}\right)^{2} \hat{v}_{\tau}^{2}\right\}
\end{aligned}
$$

with $\hat{v}_{F}^{2}=v_{F}^{2} /\left(v_{F}^{2}+a_{F}^{2}\right)$ etc.

To give a flavour of the sensitivity of $\mathrm{e}^{+} \mathrm{e}^{-}$collider data at the $Z$ we base our evaluation on the averages of published data as summarized in [17]. We use the following measurements as input to our fits: The mass of the $Z, M_{Z}=91.187 \pm 0.007 \mathrm{GeV}$, the total width of the $Z, \Gamma_{Z}=2.490 \pm 0.007 \mathrm{GeV}$, the hadronic pole cross section, $\sigma_{h}^{0}=41.55 \pm 0.14 \mathrm{nb}$, the ratio of the hadronic partial width to the partial width for $Z$ decays into electron, muon and tau pairs, $R_{e}=20.76 \pm 0.08, R_{\mu}=20.76 \pm 0.07$ and $R_{\tau}=20.80 \pm 0.08$, the forward-backward pole asymmetries for electrons, muons and taus, $A_{F B}^{0, e}=0.0151 \pm 0.040, A_{F B}^{0, \mu}=0.0133 \pm 0.0026$ and $A_{F B}^{0, \tau}=0.0212 \pm 0.0032$, the integrated tau polarization asymmetry, $\mathcal{A}_{\tau}=0.141 \pm 0.021$, the combination of the tau polarization forward-backward asymmetry and the measurement of the left-right polarization asymmetry at SLC, $\mathcal{A}_{e}=0.161 \pm 0.012$, the forward-backward pole asymmetries for $b$ and $c$ quarks, $A_{F B}^{0, b}=0.107 \pm 0.013$ and $A_{F B}^{0, c}=0.058 \pm 0.022$ and the ratio of the partial width of the $Z$ for decays into $b$ quarks to the hadronic partial width, $\Gamma_{b \bar{b}} / \Gamma_{h a d}=0.2210 \pm 0.0029$.

For the sake of simplicity we assume the radius $R$ to be independent of the particle species. For the evaluation of the Standard Model prediction we use the electroweak library provided by the program ZFITTER [18]. The mass of the top quark $m_{t}$ is treated as an additional free parameter. Also the strong coupling constant $\alpha_{s}$ is treated as free parameter within the limits $\alpha\left(m_{Z}^{2}\right)=0.123 \pm 0.006$ [19]. As the results presented below are not sensitive to a variation of the mass of the Higgs boson between 60 and $1000 \mathrm{GeV}$, this unknown parameter has been fixed to $m_{H}=300 \mathrm{GeV}$.

We find the values listed in Table 2 for the anomalous magnetic moments in the linear and quadratic $F$ mass scenarios. In the bottom part of the table we compare the results with the corresponding bounds derived from the $(g-2)$ measurements [2]. The $Z$ decay data are all compatible with a vanishing fermion radius and with no anomalous contributions to the magnetic moments.

The analysis of the heavy $\tau$ final states is of special interest in this context [13 15]. Early PETRA data have been used [14 to constrain the anomalous magnetic dipole 


\begin{tabular}{|c||c|c||c|c|}
\hline \multicolumn{1}{|c||}{} & \multicolumn{2}{c||}{$\begin{array}{c}\text { linear } F \text { mass } \\
\text { dependence of } \kappa\end{array}$} & \multicolumn{2}{c|}{$\begin{array}{c}\text { quadratic } F \text { mass } \\
\text { dependence of } \kappa\end{array}$} \\
\hline \hline$Z F \bar{F}$ & $R=(0.0 \pm 0.1) \cdot 10^{-3} f m$ & \multicolumn{2}{c|}{$R=(0.0 \pm 0.1) \cdot 10^{-3} f m$} \\
\hline & $\eta_{1} R[\mathrm{fm}]$ & $\kappa$ & $\eta_{2} R[\mathrm{fm}]$ & $\kappa$ \\
\hline $\mathrm{e}$ & $\left(-0.4_{-0.2}^{+1.1}\right) \cdot 10^{-2}$ & $\left(-0.2_{-0.1}^{+0.5}\right) \cdot 10^{-5}$ & $1.3_{-2.8}^{+0.3}$ & $\left(0.4_{-1.9}^{+0.2}\right) \cdot 10^{-6}$ \\
$\mu$ & $\left(-0.4_{-0.3}^{+1.0}\right) \cdot 10^{-2}$ & $\left(-0.4_{-0.3}^{+1.1}\right) \cdot 10^{-3}$ & $\left(0.8_{-1.9}^{+0.3}\right) \cdot 10^{-1}$ & $\left(0.7_{-3.4}^{+0.6}\right) \cdot 10^{-4}$ \\
$\tau$ & $\left(0.4_{-0.7}^{+0.3}\right) \cdot 10^{-2}$ & $\left(0.7_{-1.2}^{+0.5}\right) \cdot 10^{-2}$ & $\left(0.2_{-0.5}^{+0.1}\right) \cdot 10^{-1}$ & $\left(0.1_{-0.6}^{+0.1}\right) \cdot 10^{-2}$ \\
$u, d$ & $(0.0 \pm 0.6) \cdot 10^{-3}$ & $(0.0 \pm 0.2) \cdot 10^{-5}$ & $(0.0 \pm 0.4) \cdot 10^{-1}$ & $(0.0 \pm 0.3) \cdot 10^{-10}$ \\
$s$ & $(0.0 \pm 0.6) \cdot 10^{-3}$ & $(0.0 \pm 0.5) \cdot 10^{-4}$ & $(0.0 \pm 0.4) \cdot 10^{-1}$ & $(0.0 \pm 0.2) \cdot 10^{-7}$ \\
$c$ & $(0.0 \pm 0.2) \cdot 10^{-2}$ & $(0.0 \pm 0.1) \cdot 10^{-2}$ & $(0.0 \pm 0.2) \cdot 10^{-1}$ & $(0.0 \pm 0.4) \cdot 10^{-6}$ \\
$b$ & $(0.6 \pm 0.5) \cdot 10^{-3}$ & $(0.2 \pm 0.2) \cdot 10^{-2}$ & $\left(0.6_{-0.5}^{+0.2}\right) \cdot 10^{-2}$ & $\left(0.4_{-0.6}^{+0.2}\right) \cdot 10^{-3}$ \\
\hline \hline$\gamma \mathrm{e} \overline{\mathrm{e}}$ & \multicolumn{4}{c}{$\kappa=(0.5 \pm 0.3) \cdot 10^{-10}$} \\
$\gamma \mu \bar{\mu}$ & \multicolumn{4}{c}{$\kappa=(0.8 \pm 1.1) \cdot 10^{-8}$} \\
\hline
\end{tabular}

Table 2: Bounds on the particle radius $R$ and the anomalous magnetic moments $\kappa$. Upper part: bounds derived from $L E P Z$ decays $\left[\chi^{2} /\right.$ d.o.f. $\left.=10 / 5\right]$. Lower part: bounds from $(g-2)$ measurements; they are defined by the difference between the theoretical and experimental average values with their errors added in quadrature.

moment of the $\tau$ to less than 0.014. A bound of 0.036 may finally be obtained from $\gamma$ radiation in $\tau$ pair production of $Z$ decays [15]. It is apparent from Table 2 that the bound on the $Z$ anomalous dipole moment is stronger than these estimates.

As expected, also the bounds on the anomalous magnetic moments of the light quarks improve considerably compared to earlier evaluations of PETRA data [14,16]. The limits on the anomalous magnetic moments of the heavy $c, b$ quarks are much stronger than the bounds obtained from the low-energy data.

\section{Electron-Quark Scattering}

\section{$3.1 \quad \mathcal{N C}$ Processes}

The cross sections for electron-quark scattering $e^{ \pm}\left(\frac{-}{q}\right) \rightarrow e^{ \pm}\left(\frac{-}{q}\right)$ at HERA energies are builtup by photon and $Z$-boson exchanges. For large values of the momentum transfer, $Q^{2}$ of order $10^{4} \mathrm{GeV}^{2}$, the $Z$-exchange contributions are of the same order as the $\gamma$ contributions. Restricting ourselves to light quark targets, the cross section $e p \rightarrow e X$ can be decomposed 
into the following incoherent sum of helicity cross sections,

$$
\frac{d \sigma^{\mathcal{N C}}}{d x d Q^{2}}=\frac{4 \pi \alpha^{2}}{Q^{4}} \frac{1}{4} \sum_{q} \sum_{L, R} q\left(x, Q^{2}\right) \sigma_{i j}^{k l} f_{i j}^{k l}(y)
$$

(including quark and antiquark parton densities $q$ ). $y$ denotes the usual energy transfer variable $p q / p k_{e}$. Defining the generalized charges for vector/axial-vector and spin currents in the process $e_{i}^{-}+q_{k} \rightarrow e_{j}^{-}+q_{l}$ as

$$
\begin{array}{ll}
Q_{i}^{k}=e_{e} e_{q}+\frac{G_{F} m_{Z}^{2}}{8 \sqrt{2} \pi \alpha} \epsilon_{e}^{i} \epsilon_{q}^{k} \frac{Q^{2}}{Q^{2}+m_{Z}^{2}} & \text { for } V_{e} \times V_{q} \\
Q_{i}^{\prime}=e_{e} e_{q}+\frac{G_{F} m_{Z}^{2}}{8 \sqrt{2} \pi \alpha} \epsilon_{e}^{i} v_{q} \frac{Q^{2}}{Q^{2}+m_{Z}^{2}} & \text { for } V_{e} \times \Sigma_{q} \\
Q^{\prime k}=e_{e} e_{q}+\frac{G_{F} m_{Z}^{2}}{8 \sqrt{2} \pi \alpha} v_{e} \epsilon_{q}^{k} \frac{Q^{2}}{Q^{2}+m_{Z}^{2}} & \text { for } \Sigma_{e} \times V_{q} \\
Q^{\prime \prime}=e_{e} e_{q}+\frac{G_{F} m_{Z}^{2}}{8 \sqrt{2} \pi \alpha} v_{e} v_{q} \frac{Q^{2}}{Q^{2}+m_{Z}^{2}} & \text { for } \Sigma_{e} \times \Sigma_{q}
\end{array}
$$

the coefficients $\sigma_{i j}^{k l}$ are given by

$$
\sigma_{i j}^{k l}= \begin{cases}\left|Q_{i}^{k}\right|^{2} f_{e}^{2} f_{q}^{2} & \text { for } V_{e} \times V_{q} \\ \left|Q_{i}^{\prime}\right|^{2} f_{e}^{2} \frac{1}{4}\left(\kappa_{q} / m_{q}\right)^{2} s_{e q} & \text { for } V_{e} \times \Sigma_{q} \\ \left|Q^{\prime k}\right|^{2} \frac{1}{4}\left(\kappa_{e} / m_{e}\right)^{2} s_{e q} f_{q}^{2} & \text { for } \Sigma_{e} \times V_{q} \\ \left|Q^{\prime \prime}\right|^{2} \frac{1}{16}\left(\kappa_{e} / m_{e}\right)^{2}\left(\kappa_{q} / m_{q}\right)^{2} s_{e q}^{2} & \text { for } \Sigma_{e} \times \Sigma_{q}\end{cases}
$$

where $s_{e q}$ denotes the invariant energy squared of the $e q$ subprocess. The $y$ dependent coefficients are related to the scattering angle, $y=\left(1+\cos \theta_{*}\right) / 2$, in the $(e q)$ c.m. frame,

$$
f_{i j}^{k l}(y)= \begin{cases}1 & \text { for } V_{e} \times V_{q} \text { and equal } e / q \text { helicities } \\ (1-y)^{2} & \text { for } V_{e} \times V_{q} \text { and opposite } e / q \text { helicities } \\ y(1-y) & \text { for } V_{e} \times \Sigma_{q} \text { and } v . v \\ y^{2}\left(1-\frac{1}{2} y\right)^{2} & \text { for } \Sigma_{e} \times \Sigma_{q}\end{cases}
$$

Switching from electrons to positrons or/and quarks to antiquarks, the helicity factors $f$ remain the same while the generalized charges of the antiparticles are to be identified with the negative charges of the particles, yet the helicity indices reversed. The first two entries in eq.(14) are well-known consequences of angular momentum conservation for helicity-conserving vector/axial-vector couplings. Since the spin couplings flip the helicities, the interference term between $V$ and $\Sigma$ couplings must vanish for forward and backward scattering; this condition is met by the coefficient $y(1-y)=\left(1-\cos ^{2} \theta_{*}\right) / 4$. Fierzing the $\Sigma \times \Sigma$ amplitude from $t$ to $s$-channel exchange amplitudes results in a mixture 
of spin-0 and spin-1 amplitudes so that no simple angular pattern can be derived in this case.

Note that the contributions due to the anomalous magnetic moments come with characteristic $y(1-y)$ and $y^{2}\left(1-\frac{1}{2} y\right)^{2}$ coefficients which are different from the familiar $\left[1 \pm(1-y)^{2}\right]$ coefficients of the leading $V, A$ currents and the $y^{2}$ coefficient of the non-leading longitudinal structure function. Non-zero quark radii, on the other hand, affect the $Q^{2}$ dependence of the deep inelastic cross section in a characteristic way.

Assuming that the cross section $d \sigma(e P \rightarrow e X) / d Q^{2}$ can be measured at HERA with an accuracy of order $1 \%$ for $Q^{2}$ values of order $10^{3} \mathrm{GeV}^{2}$, the radius of the light quarks can be limited to $R_{q} \lesssim 10^{-16} \mathrm{~cm}$. Note that the $\gamma$ exchange dominates the cross section in this $Q^{2}$ range. This bound is therefore complementary to the bound extracted from $Z$ decay data, and can truly be associated with the quark radius. If the anomalous $y$ dependent terms can also be limited to a level of $1 \%$, the $\kappa_{q}$ parameter for quarks is restricted to less than $\kappa_{q} \lesssim 10^{-4}$ for light quarks $u, d$.

\section{$3.2 \quad \mathcal{C C}$ Processes}

The cross sections for the charged current processes $e^{ \pm}\left(\frac{-}{q}\right) \rightarrow\left(-\frac{-}{\nu}\right)\left(-\frac{1}{q}\right)$ can be written in a similar compact form,

$$
\frac{d \sigma^{\mathcal{C C}}}{d x d Q^{2}}=\frac{G_{F}^{2}}{2 \pi}\left[\frac{m_{W}^{2}}{Q^{2}+m_{W}^{2}}\right]^{2} \sum_{q} \sum_{L, R} q\left(x, Q^{2}\right) \sigma_{i L}^{k L} f_{i L}^{k L}(y)
$$

If only the left-handed neutrinos and final state quarks contribute to the coefficients $\sigma_{i j}^{k l}$ $[j=l=L]$ of $\sigma\left(e_{i}^{ \pm} q_{k} \rightarrow \nu_{j} q_{l}^{\prime}\right)$, we obtain

$$
\sigma_{i L}^{k L}= \begin{cases}f_{e}^{2} f_{q}^{2} & \text { for } V_{e} \times V_{q} \\ f_{e}^{2} \frac{1}{4}\left(\kappa_{q} / m_{q}\right)^{2} s_{e q} & \text { for } V_{e} \times \Sigma_{q} \\ \frac{1}{4}\left(\kappa_{e} / m_{e}\right)^{2} s_{e q} f_{q}^{2} & \text { for } \Sigma_{e} \times V_{q} \\ \frac{1}{16}\left(\kappa_{e} / m_{e}\right)^{2}\left(\kappa_{q} / m_{q}\right)^{2} s_{e q}^{2} & \text { for } \Sigma_{e} \times \Sigma_{q}\end{cases}
$$

The $y$ dependent coefficients agree with the expressions in eq.(14).

First measurements of the $\mathcal{C C}$ cross section at HERA have been carried out recently [20]. Bounds on the radii and the transition magnetic moments of the quarks from $\mathcal{C C}$ processes are expected in the same range as for $\mathcal{N C}$ processes when the statistics at HERA will grow in the years to come. 


\section{Summa}

\begin{tabular}{|c||cc|}
\hline$R[\mathrm{fm}]$ & $\begin{array}{c}\text { linear mass } \\
\text { dependence of } \kappa\end{array}$ & $\begin{array}{c}\text { quadratic mass } \\
\text { dependence of } \kappa\end{array}$ \\
\hline \hline$Z F \bar{F}$ & $(0.0 \pm 0.1) \cdot 10^{-3}$ & $(0.0 \pm 0.1) \cdot 10^{-3}$ \\
\hline$\gamma e \bar{e}$ & $(0.2 \pm 0.1) \cdot 10^{-7}$ & $(0.3 \pm 0.1) \cdot 10^{-2}$ \\
$\gamma \mu \bar{\mu}$ & $(0.1 \pm 0.2) \cdot 10^{-7}$ & $(0.2 \pm 0.1) \cdot 10^{-3}$ \\
\hline
\end{tabular}

Table 3: Bounds on the radii of the particles in the Standard Model, derived from the LEP Z experiments and the $(g-2)_{e, \mu}$ measurements. Bounds on anomalous magnetic dipole moments are converted into bounds on radii $R$ by setting $\kappa_{F}=m_{F} R$ and $\left(m_{F} R\right)^{2}$ for the first and second column, respectively. For the fit to the $Z$ data $\chi^{2} /$ d.o.f. $=12 / 11$.

We may summarize the results presented in the previous sections by setting $\eta_{1}$ and $\eta_{2}=1$ and converting the bounds on the anomalous dipole moments into bounds on the radius $R$ of the fermions. The results on $R$ are collected in Table 3 for the high energy $Z$ decay experiments. They are compared with the values of $R$ for electrons and muons derived from $(g-2)$ experiments in the same way. In the quadratic $F$ mass scenario the bounds on the radii of leptons and quarks from $Z$ decays are competitive with the bounds derived from the $(g-2)_{\mu}$ experiments. In this scenario fermions appear to have radii

$$
R \lesssim 10^{-17} \mathrm{~cm}
$$

HERA is expected to set bounds of similar size on quarks and electrons in the electromagnetic and weak sectors separately.

Acknowledgement. We are grateful to S.J.Brodsky for valuable discussions and comments on the manuscript.

\section{REFERENCES}

[1] M.Chanowitz and S.D.Drell, Phys.Rev.Lett. 30 (1973) 807; G.B.West and P.M.Zerwas, Phys.Rev. D10 (1974) 2130.

[2] See e.g. the review articles by T.Kinoshita and D.R.Yennie, and R.S.van Dyck Jr. in "Quantum Electrodynamics", ed. T.Kinoshita, World Scientific 1990. 
[3] S.J.Brodsky and S.D.Drell, Phys.Rev. D22 (1980) 2236.

[4] S.J.Brodsky and F.Schlumpf, Phys.Lett. B329 (1994) 111.

[5] S.J.Brodsky and J.R.Hiller, Phys.Rev. D46 (1992) 2141.

[6] E.Eichten, K.Lane and M.E.Peskin, Phys.Rev.Lett. 50 (1983) 811;

B.Schrempp, F.Schrempp, N.Wermes and D.Zeppenfeld, Nucl.Phys. B296 (1988) 1.

[7] H.Kroha, Phys.Rev. D46 (1992) 58.

[8] F.Abe et al., Phys.Rev.Lett. 71 (1993) 2542.

[9] See e.g. W.Bernreuther, O.Nachtmann and P.Overmann, Phys.Rev. D48 (1993) 78;

B.Ananthanarayan and S.D.Rindani, Phys.Rev.Lett. 73 (1994) 1215;

C.A.Nelson, Report SUNY BING 4/30/94;

and earlier references quoted in these papers.

[10] P.D.Acton et al., Phys.Lett. B281 (1992) 405;

D.Buskulic et al., Phys.Lett. B297 (1992) 459.

[11] J.Gasser and H.Leutwyler, Phys.Rep. 87 (1982) 77.

[12] A.Djouadi in " $e^{+} e^{-}$Collisions at $500 \mathrm{GeV}$ : The Physics Potential", ed. P.M.Zerwas, DESY 92-123B.

[13] A.Djouadi and M.Spira, Phys.Lett. B228 (1989) 443.

[14] D.J.Silverman and G.L.Shaw, Phys.Rev. D27 (1983) 1196.

[15] J.A.Grifols and A.Mendez, Phys.Lett. B255 (1991) 611.

[16] S.Fajfer and R.J.Oakes, Phys.rev. D28 (1983) 2881.

[17] Particle Data Group, Phys. Rev. D50 (1994) 1173.

[18] Line shape program ZFITTER, version 4.6, Dubna-Zeuthen Radiative Correction Group; D. Bardin et al., Comp. Phys. Comm. 59 (1990) 303; Z. Phys. C44 (1989) 493; Nucl. Phys. B351 (1991) 1; Phys. Lett. B229 (1989) 405; CERN-TH 6443/92.

[19] S.Bethke, Proceedings, Linear Collider Workshop, Waikoloa/Hawaii, 1993;

S. Catani, Proceedings, International Europhysics Conference, Marseille, 1993;

S. Banerjee, ibid.

[20] T.Ahmed et al., Phys.Lett. B324 (1994) 241. 